\title{
ABSTRACT NASH MANIFOLDS
}

\author{
M. SHIOTA
}

\begin{abstract}
We prove that any abstract noncompact Nash manifold is $C^{\infty}$ diffeomorphic to the interior of some compact $C^{\infty}$ manifold with boundary, and conversely, that such an interior or a compact $C^{\infty}$ manifold admits infinitely many abstract Nash manifold structures. The last result is a generalization of [2], where the case of a torus is proved.
\end{abstract}

1. Introduction. A continuous map of semialgebraic sets is called semialgebraic if the graph is semialgebraic. Let $\Gamma$ denote the pseudogroup of all semialgebraic analytic diffeomorphisms between semialgebraic open subsets of a Euclidean space. An abstract Nash manifold is a manifold with $\Gamma$-structure and with a finite system of coordinate neighborhoods. An analytic map $f: M_{1} \rightarrow M_{2}$ of abstract Nash manifolds is called a Nash map if for each point $x \in M_{1}$, there exist coordinate neighborhoods $h_{1}: U_{1} \rightarrow \mathbf{R}^{n}$ and $h_{2}: U_{2} \rightarrow \mathbf{R}^{m}$ of $x, f(x)$ in $M_{1}, M_{2}$ respectively, such that $h_{2} \circ f \circ h_{1}^{-1}: h_{1}\left(U_{1} \cap f^{-1}\left(U_{2}\right)\right) \rightarrow \mathbf{R}^{m}$ is semialgebraic. If an abstract Nash manifold has a Nash imbedding in some Euclidean space, we call it an affine Nash manifold. We can define the above concepts by terms familiar to algebraists (see [1] and [2]). In this paper, manifolds do not have boundary unless otherwise specified.

Affine Nash manifold structures on $C^{\infty}$ manifolds were studied and clarified by Nash [5] and the author [7]. To be precise, a compact $C^{\infty}$ manifold admits the unique compatible affine Nash manifold structure [5], and this holds true even if the manifold has boundary [7]. For each noncompact affine Nash manifold $M$, there exists uniquely up to Nash diffeomorphism a compact affine Nash manifold with boundary whose interior is Nash diffeomorphic to $M[\mathbf{7}]$; in a word, $M$ admits a unique compactification.

We know little about abstract Nash manifolds. On $S^{1}$, Mazur [6] showed a nonaffine abstract Nash manifold structure. Moreover, Chillingworth, Hubbard, and Mazur [2] proved that any torus admits infinitely many distinct abstract Nash manifold structures. We want to generalize these results. A $C^{\infty}$ manifold is called compactifiable if it is $C^{\infty}$ diffeomorphic to the interior of some compact $C^{\infty}$ manifold with boundary.

THEOREM 1. Each abstract Nash manifold is compact or compactifiable as a $C^{\infty}$ manifold.

THEOREM 2. Any compact or compactifiable $C^{\infty}$ manifold admits infinitely many compatible nonaffine abstract Nash manifold structures.

Received by the editors May 3, 1984 and, in revised form, February 20, 1985.

1980 Mathematics Subject Classification. Primary 58A07; Secondary 14G30.

Key words and phrases. Nash manifold, real algebraic geometry, semialgebraic set. 
In $\S 2$ we introduce the concept of a $C^{r}$ Nash manifold for $0<r<\infty$ and prove that any abstract $C^{r}$ Nash manifold is affine. As a consequence, we obtain Theorem 1. See [8] for detailed properties of a $C^{0}$ Nash manifold structure.

2. $C^{r}$ Nash manifold. Let $0<r<\infty$. Replacing "analytic" in $\S 1$ by " $C$ ", we define an abstract $C^{r}$ Nash manifold.

REMARK 2.1. If we define a $C^{r}$ Nash map in the same way as in $\S 1$, then a Nash map of Euclidean spaces is locally semialgebraic but not necessarily semialgebraic. For example, $f(x)=(x-[x]-1)^{r+1}(x-[x])^{r+1}$, where [ ] denotes the Gauss symbol. Such a function holds no interesting global property because it is not "of finite type".

REMARK 2.2. Let $f: M_{1} \rightarrow M_{2}$ be a Nash map from an abstract Nash manifold to an affine one. Then, for each pair $h_{1}: U_{1} \rightarrow \mathbf{R}^{n}$ and $h_{2}: U_{2} \rightarrow \mathbf{R}^{m}$ of coordinate neighborhoods of $M_{1}$ and $M_{2}, h_{1}\left(U_{1} \cap f^{-1}\left(U_{2}\right)\right)\left(\subset \mathbf{R}^{n}\right)$ and $h_{2} \circ f \circ h_{1}^{-1}$ : $h_{1}\left(U_{1} \cap f^{-1}\left(U_{2}\right)\right) \rightarrow \mathbf{R}^{m}$ are semialgebraic. The proof below of Theorem 2 shows that it is not necessarily the case for general $M_{2}$.

ProOF OF REMARK 2.2. First consider the case $M_{1}=U_{1} \subset \mathbf{R}^{n}, M_{2}=U_{2} \subset$ $\mathbf{R}^{m}$, and $h_{1}=h_{2}=$ ident. Because the graph of $f$ is closed in $U_{1} \times \mathbf{R}^{m}$ and consists of a finite number of connected components, it suffices to prove the following. Given an open semialgebraic set $W \subset \mathbf{R}^{l}$ and a closed subset $X \subset W$, assume that $X$ is a connected analytic manifold and that each $x \in X$ has a semialgebraic neighborhood in $X$ (i.e., $X$ is locally semialgebraic). Then $X$ is semialgebraic. Indeed, let $V$ be a semialgebraic open subset of $X$. Let $g_{1}, \ldots, g_{k}$ be generators of the ideal of $V$ in the polynomial ring on $\mathbf{R}^{l}$. Then they vanish on $X$ since $X$ is a connected analytic manifold. Let $Y$ be the set of common zeros and $Z$ be the set of $y \in Y$ where some neighborhoods of $y$ in $Y$ are analytic manifolds of dimension $=\operatorname{dim} X$. Then $Z \cap W$ is a semialgebraic analytic manifold and hence consists of a finite number of connected components, and $Y-Z$ is closed in $\mathbf{R}^{l}$ and is of dimension $<\operatorname{dim} X$, e.g. [2]. Because $X \cap Z$ is an analytic manifold that is closed in $W-(Y-Z)$ and contained in $Z \cap W$, it is a union of some connected components of $Z \cap W$. Hence, $X \cap Z$ is semialgebraic [2]. It follows from $\operatorname{dim}(Y-Z)<\operatorname{dim} X$ that the closure of $X \cap Z$ in $W$ is $X$, which proves that $X$ is semialgebraic.

The case $M_{2}=\mathbf{R}^{m}$ follows immediately from the proof above. For the general case let $\phi: M_{2} \rightarrow \mathbf{R}^{l}$ be a Nash imbedding. Then $\phi \circ f: M_{1} \rightarrow \mathbf{R}^{l}$ is a Nash map. Hence by the second case, $\phi \circ f \circ h_{1}^{-1}: h_{1}\left(U_{1}\right) \rightarrow \mathbf{R}^{l}$ is semialgebraic. The second case also implies that $\phi \circ h_{2}^{-1}: h_{2}\left(U_{2}\right) \rightarrow \mathbf{R}^{l}$ is semialgebraic, which shows that $\phi\left(U_{2}\right)=\phi \circ h_{2}^{-1}\left(h_{2}\left(U_{2}\right)\right) \subset \mathbf{R}^{l}$ is semialgebraic. Remark 2.2 follows from these, because

$$
\left(\phi \circ f \circ h_{1}^{-1}\right)^{-1}\left(\phi\left(U_{2}\right)\right)=h_{1}\left(U_{1} \cap f^{-1}\left(U_{2}\right)\right)
$$

and because the graph of $h_{2} \circ f \circ h_{1}^{-1}: h_{1}\left(U_{1} \cap f^{-1}\left(U_{2}\right)\right) \rightarrow \mathbf{R}^{m}$ is the image of

$$
\left\{(x, y, z) \in h_{1}\left(U_{1}\right) \times h_{2}\left(U_{2}\right) \times \mathbf{R}^{l} \mid z=\phi \circ f \circ h_{1}^{-1}(x)=\phi \circ h_{2}^{-1}(y)\right\}
$$

under the projection $h_{1}\left(U_{1}\right) \times h_{2}\left(U_{2}\right) \times \mathbf{R}^{l} \rightarrow h_{1}\left(U_{1}\right) \times h_{2}\left(U_{2}\right)$.

Let $f: M_{1} \rightarrow M_{2}$ be a $C^{r}$ map of abstract $C^{r}$ Nash manifolds. If $h_{1}\left(U_{1} \cap f^{-1}\left(U_{2}\right)\right)$ and $h_{2} \circ f \circ h_{1}^{-1}: h_{1}\left(U_{1} \cap f^{-1}\left(U_{2}\right)\right) \rightarrow \mathbf{R}^{m}$ are semialgebraic for each pair $h_{1}: U_{1} \rightarrow \mathbf{R}^{n}$ and $h_{2}: U_{2} \rightarrow \mathbf{R}^{m}$ of coordinate neighborhoods of $M_{1}$ and $M_{2}$, then we call $f$ a $C^{r}$ Nash map. An affine $C^{r}$ Nash manifold is an abstract 
$C^{r}$ Nash manifold with a $C^{r}$ Nash imbedding in some Euclidean space. A property of $C^{r}$ Nash manifolds completely different from Nash manifolds is the following.

THEOREM 2.3. An abstract $C^{r}$ Nash manifold is affine.

Proof. Let $M$ be an abstract $C^{r}$ Nash manifold, and $h_{i}: U_{i} \rightarrow \mathbf{R}^{n}, i=1, \ldots, k$, be a system of coordinate neighborhoods of $M$. By a theorem of Mostowski [4], there are semialgebraic functions $\phi_{i}, i=1, \ldots, k$, on $\mathbf{R}^{n}$ such that $\phi_{i}^{-1}(0)=$ $\overline{h_{i}\left(U_{i}\right)}-h_{i}\left(U_{i}\right)$ and $\left.\phi_{i}\right|_{h_{i}\left(U_{i}\right)}$ are Nash functions. For each $i$, set $h_{i}^{\prime}=\left(h_{i}, 1 / \phi_{i}\right.$ 。 $\left.h_{i}\right)$. Then $h_{i}^{\prime}$ is a proper $C^{r}$ Nash imbedding of $U_{i}$ in $\mathbf{R}^{n+1}$. As $\mathbf{R}^{n+1}$ is Nash diffeomorphic to $S^{n+1}$-a point by the stereographic projection, $h_{i}^{\prime}$ induces a $C^{r}$ Nash imbedding $h_{i}^{\prime \prime}: U_{i} \rightarrow \mathbf{R}^{n+2}$ such that $h_{i}^{\prime \prime}\left(U_{i}\right)$ is bounded and that $\overline{h_{i}^{\prime \prime}\left(U_{i}\right)}-$ $h_{i}^{\prime \prime}\left(U_{i}\right)$ is a point. Assume the point $=0$. Set

$$
\psi\left(x_{1}, \ldots, x_{n+2}\right)=\sum_{j=1}^{n+2} x_{j}^{2 l} \times\left(x_{1}, \ldots, x_{n+2}\right), \quad g_{i}=\psi \circ h_{i}^{\prime \prime}
$$

for a sufficiently large integer $l$. Then $g_{i}$ holds the same properties as $h_{i}^{\prime \prime}$; moreover, Lojasiewicz' inequality says that we can extend $g_{i}$ to $M$ as a $C^{r}$ Nash map by defining $g_{i}=0$ on $M-U_{i}$. Hence, $g: \prod_{i=1}^{k} g_{i}: M \rightarrow \mathbf{R}^{k(n+2)}$ is a $C^{r}$ Nash imbedding.

THEOREM 2.4. A $C^{r}$ Nash manifold is compact or compactifiable as a $C^{r}$ manifold.

Proof. Let $M$ be a noncompact $C^{r}$ Nash manifold. By the proof of Theorem 2.3 there is a bounded $C^{r}$ Nash imbedding $g: M \rightarrow \mathbf{R}^{m}$ such that $\overline{g(M)}-g(M)$ is a point, say 0 . Hence, we can assume that $M$ is a bounded subset of $\mathbf{R}^{n}$ satisfying $\bar{M}-M=0$. We note that $\bar{M}$ is semialgebraic. Recall the well-known fact (e.g. [2]) that a semialgebraic set admits a Whitney stratification, from which it follows that the tangent space $T_{x} M$ is not perpendicular to the vector $0 x$, for $x \in M$ near 0 . Let $v_{x}$ be the orthogonal projection image of $x 0$ to $T_{x} M$. Then $v$ is a $C^{r-1}$ nonsingular vector field on $M$ around 0 . Assume $r \geq 2$. Then, integrating $v$ we obtain a $C^{r-1}$ diffeomorphism from $M \cap\{|x| \leq \varepsilon\}$ to $(M \cap\{|x|=\varepsilon\}) \times(0,1]$ for small $\varepsilon>0$. Here, $M \cap\{|x|=\varepsilon\}$ is a compact $C^{r}$ manifold, and hence we can approximate the $C^{r-1}$ diffeomorphism by a $C^{r}$ one. Therefore, $M$ is compactifiable. If $r=1$, consider a close approximation $M^{\prime}$ of $M$ by a $C^{\infty}$ manifold in the Whitney $C^{1}$ topology such that $M^{\prime}$ and $M$ are $C^{1}$ diffeomorphic and $M^{\prime}$ remains to satisfy the conditions on $M$ (i.e., $\bar{M}^{\prime}-M^{\prime}=0$ and $\left(M^{\prime}, 0\right)$ satisfies the Whitney condition). Then, by the above argument, $M^{\prime}$ is compactifiable as a $C^{\infty}$ manifold. Hence, $M$ is compactifiable as a $C^{\mathbf{1}}$ manifold. Thus, the theorem is proved.

ProOF OF THEOREM 1. Trivial by Theorem 2.4 .

3. Proof of Theorem 2. Let $M$ be a compact or compactifiable $C^{\infty}$ manifold. We will construct nonaffine abstract Nash manifolds $M_{c}$ parametrized by $0<c<1$ such that

(3.1) $M_{c}$ are diffeomorphic to $M$, and 
(3.2) $M_{c}$ and $M_{c^{\prime}}$ are not Nash diffeomorphic if $\log c / \log c^{\prime}$ is irrational. Let $c$ be fixed, and set

$$
\begin{aligned}
& a=2^{5 / 2}(1+c)^{2} /(1-c)^{2}, \\
& d=2+2^{3 / 2} a-\left(a+2^{1 / 2}\right)\left(-a+\left(a^{2}+2^{5 / 2} a\right)^{1 / 2}\right) .
\end{aligned}
$$

We easily see that $2^{5 / 2}<a$ and $1<d<2$.

I. First consider the case $M=\mathbf{R}$. Set

$$
N_{1}=(-\infty, d), \quad N_{2}=(0, \infty), \quad N_{3}=(0,1) .
$$

Let $f$ be a Nash function on $N_{3}$ satisfying

$$
2^{1 / 2}(x+f(x))=(x-f(x))^{2} / a .
$$

If we rotate the graph of $f$ with center at 0 , then it comes to the graph of $y=x^{2} / a$. From this, and from the inequality $2^{5 / 2}<a$ it follows that $f$ and its Nash extension $\tilde{f}$ to $\left[1-2^{-1 / 4} a^{1 / 2}, 1+2^{-1 / 4} a^{1 / 2}\right]$ is well defined so that

$$
\tilde{f}\left[1-2^{-1 / 4} a^{1 / 2}, 1+2^{-1 / 4} a^{1 / 2}\right]=\left[1-2^{-1 / 4} a^{1 / 2}, 1+2^{-1 / 4} a^{1 / 2}\right],
$$

the derivative of $\tilde{f}<0$, and $\tilde{f} \circ \tilde{f}=$ ident. Define Nash maps $h_{1}: N_{3} \rightarrow N_{1}$ and $h_{2}: N_{3} \rightarrow N_{2}$ by

$$
h_{1}(x)=x^{2}+f^{2}(x) \text { and } h_{2}(x)=-x^{2}+2 x .
$$
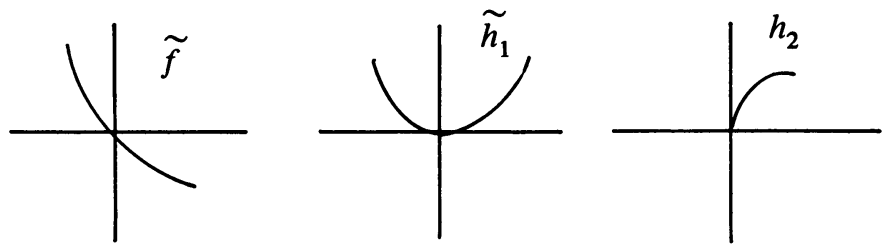

Then there are Nash imbeddings such that $h_{1}\left(N_{3}\right)=(0, d), h_{2}\left(N_{3}\right)=(0,1)$. Note that $h_{1}$ can be extended to $\tilde{h}_{1}:\left[1-2^{-1 / 4} a^{1 / 2}, 1+2^{-1 / 4} a^{1 / 2}\right] \rightarrow \mathbf{R}$ as a Nash function whose derivative vanishes only at 0 and satisfies $\tilde{h}_{1}(x)=\tilde{h}_{1}(\tilde{f}(x))$ because the derivative of $\tilde{f}<0$ and $\tilde{f} \circ \tilde{f}=$ ident. Consider the disjoint union of $N_{1}, N_{2}$, and $N_{3}$, and an equivalence relation $x \sim h_{1}(x)$ and $x \sim h_{2}(x)$ on the union. Then the quotient topological space admits an abstract Nash manifold structure induced by those of $N_{1}, N_{2}$, and $N_{3}$. Let $M_{c}$ denote the abstract Nash manifold. Trivially (3.1).

Put $\bar{N}_{3}=[0,1]$ and consider it to contain $N_{3}$. Let $\rho: \bar{N}_{3} \rightarrow M_{c}$ be the natural map. To prove (3.2), we use the Nash map

$$
\phi:\left(1-2^{-1 / 4} a^{1 / 2}, 1+2^{-1 / 4} a^{1 / 2}\right) \rightarrow \rho\left(\bar{N}_{3}\right) \subset M_{c} .
$$

Define $\phi$ on $(0,1)$ so that $\rho^{-1} \circ \phi=$ ident, and extend it as widely as possible. By the method of patching of $N_{2}$ and $N_{3}$, first $\phi$ is extended to $(0,2)$ so that $\rho^{-1} \circ \phi(x)=2-x$ for $x \in[1,2)$, and 1 is the only and nondegenerate critical point. Secondly, the patching of $N_{1}$ and $N_{3}$ says that $\phi$ can be defined on $(\tilde{f}(1), 0]$ 
satisfying $\rho^{-1} \circ \phi(x)=\tilde{f}(x)$ for $x \in(\tilde{f}(1), 0)$, and its critical point is only 0 and nondegnerate. Repeating these extensions, we have

$$
\rho^{-1} \circ \phi(x)= \begin{cases}\vdots & \\ \tilde{f}(2-\tilde{f}(2-x)) & \text { on }[2-\tilde{f}(2), 2-\tilde{f}(2-\tilde{f}(1))], \\ 2-\tilde{f}(2-x) & \text { on }[2-\tilde{f}(1), 2-\tilde{f}(2)], \\ \tilde{f}(2-x) & \text { on }[2,2-\tilde{f}(1)] \\ 2-x & \text { on }[1,2] \\ x & \text { on }[0,1] \\ \tilde{f}(x) & \text { on }[\tilde{f}(1), 0] \\ 2-\tilde{f}(x) & \text { on }[\tilde{f}(2), \tilde{f}(1)], \\ \tilde{f}(2-\tilde{f}(x)) & \text { on }[\tilde{f}(2-\tilde{f}(1)), \tilde{f}(2)] \\ \vdots & \end{cases}
$$

It is seen that $\phi$ is finally defined on $\left(1-2^{-1 / 4} a^{1 / 2}, 1+2^{-1 / 4} a^{1 / 2}\right)$, that for any $e \in \bar{N}_{3}$,

$$
\begin{aligned}
\left(\rho^{-1} \circ \phi\right)^{-1}(e)=\{\ldots, 2-\tilde{f}(2-\tilde{f}(e)), 2-\tilde{f}(2-e), 2-\tilde{f}(e), 2-e, e \\
\tilde{f}(e), \tilde{f}(2-e), \tilde{f}(2-\tilde{f}(e)), \tilde{f}(2-\tilde{f}(2-e)), \ldots\}
\end{aligned}
$$

which is in order from the largest to the smallest, and that the set of critical points of $\phi$ is $\left(\rho^{-1} \circ \phi\right)^{-1}(0) \cup\left(\rho^{-1} \circ \phi\right)^{-1}(1)$ and consists of only nondegenerate ones. Place $\left(\rho^{-1} \circ \phi\right)^{-1}(e)$ in order as $\cdots<e_{i}<e_{i+1}<\ldots, i \in \mathbf{Z}$. Then we have

$$
\left(1+2^{-1 / 4} a^{1 / 2}-e_{i+2}\right) /\left(1+2^{-1 / 4} a^{1 / 2}-e_{i}\right)
$$

and

$$
\left(1-2^{-1 / 4} a^{1 / 2}-e_{i-2}\right) /\left(1-2^{-1 / 4} a^{1 / 2}-e_{-i}\right)
$$

converge to $c$ as $i \rightarrow \infty$. Because the map $x \rightarrow \tilde{f}(2-x)$ has fixed points only at the end points of the interval, it repels from $1+2^{-1 / 4} a^{1 / 2}$ and attracts to $1-2^{-1 / 4} a^{1 / 2}$ and its derivative at the latter point is $c$. Hence,

$$
\left(1-2^{-1 / 4} a^{1 / 2}-e_{-i-2}\right) /\left(1-2^{-1 / 4} a^{1 / 2}-e_{-i}\right)
$$

approaches $c$ since $e_{-i-2}=\tilde{f}\left(2-e_{-i}\right)$. A similar analysis of the map $x \rightarrow 2-\tilde{f}(x)$ shows the other convergence result.

Hence, it follows from Remark 2.2 that $M_{c}$ is not affine because a semialgebraic set consists of a finite number of connected components. We will prove (3.2) by reduction to absurdity. Assume a Nash diffeomorphism $g: M_{c} \rightarrow M_{c^{\prime}}$ for $0<c$, $c^{\prime}<1$, with irrational $\alpha=\log c^{\prime} / \log c$. Put

$$
a=2^{5 / 2}(1+c)^{2} /(1-c)^{2}, \quad a^{\prime}=2^{5 / 2}\left(1+c^{\prime}\right) /\left(1-c^{\prime}\right)^{2} .
$$

To distinguish the maps $\rho$ to $M_{c}$ and $M_{c^{\prime}}$, we write them as $\rho_{c}: N_{3} \rightarrow M_{c}$ and $\rho_{c^{\prime}}: N_{3} \rightarrow M_{c^{\prime}}$. Note that the images of $N_{1}$ and $N_{2}$, in $M_{c}$ and $M_{c^{\prime}}$, are the largest affine connected Nash submanifolds in $M_{c}$ and $M_{c^{\prime}}$ for the following reason. If it were not so, there would exist an affine connected Nash manifold which is contained in $M_{c}$ or $M_{c^{\prime}}$, and contains $\rho_{c}\left(N_{3}\right)$ or $\rho_{c^{\prime}}\left(N_{3}\right)$ respectively. But that is impossible by the above argument that says $M_{c}$ and $M_{c^{\prime}}$ are not affine. Hence 
we have $g\left(\rho_{c}\{0,1\}\right)=\rho_{c^{\prime}}\{0,1\}$. Hence $\phi_{c^{\prime}}$ and $g \circ \phi_{c}$ have the same image $=$ $\rho_{c^{\prime}}[0,1]$ and the same set of critical values $=\rho_{c^{\prime}}\{0,1\}$. Furthermore, their values vibrate infinitely from $\rho_{c^{\prime}}(0)$ to $\rho_{c^{\prime}}(1)$ and from $\rho_{c^{\prime}}(1)$ to $\rho_{c^{\prime}}(0)$. Hence, for any pair $e_{0} \in\left(1-2^{-1 / 4} a^{1 / 2}, 1+2^{-1 / 4} a^{1 / 2}\right)$ and $e_{0}^{\prime} \in\left(1-2^{-1 / 4} a^{1 / 2}, 1+2^{-1 / 4} a^{1 / 2}\right)$ with $\phi_{c^{\prime}}\left(e_{0}^{\prime}\right)=g \circ \phi\left(e_{0}\right)$, there exists a homeomorphism

$$
\tau:\left(1-2^{-1 / 4} a^{1 / 2}, 1+2^{-1 / 4} a^{1 / 2}\right) \rightarrow\left(1-2^{-1 / 4} a^{1 / 2}, 1+2^{-1 / 4} a^{1 / 2}\right)
$$

such that $\tau\left(e_{0}\right)=e_{0}^{\prime}$ and $\phi_{c^{\prime}} \circ \tau=g \circ \phi_{c}$ on $\left(1-2^{-1 / 4} a^{1 / 2}, 1+2^{-1 / 4} a^{1 / 2}\right)$. It is clear that $\tau$ is locally semialgebraic and hence semialgebraic by Remark 2.2. Recall that all critical points of $\phi_{c}$ and $\phi_{c^{\prime}}$ are nondegenerate, which proves that $\tau$ is of class $C^{\infty}$. Therefore, $\tau$ is a Nash diffeomorphism. Set

$$
\begin{aligned}
\rho_{c}^{-1} \circ \phi_{c}\left(e_{0}\right)=e, & \left(\rho_{c}^{-1} \circ \phi_{c}\right)^{-1}(e)=\left\{\cdots<e_{i}<e_{i+1}<\cdots\right\}, \\
\rho_{c^{\prime}}^{-1} \circ \phi_{c^{\prime}}\left(e_{0}^{\prime}\right)=e^{\prime}, & \left(\rho_{c^{\prime}}^{-1} \circ \phi_{c^{\prime}}\right)^{-1}\left(e^{\prime}\right)=\left\{\cdots<e_{i}^{\prime}<e_{i+1}^{\prime}<\cdots\right\} .
\end{aligned}
$$

Then $\tau$ satisfies $\tau\left(e_{i}\right)=e_{i}^{\prime}$ for any $i \in \mathbf{Z}$, or $\tau\left(e_{i}\right)=e_{-i}^{\prime}$ for any $i \in \mathbf{Z}$. For simplicity, suppose that the first equality holds and that $e_{i}$ and $e_{i}^{\prime}$ converge to 0 as $i \rightarrow-\infty$ after translating $\left(1-2^{-1 / 4} a^{1 / 2}, 1+2^{-1 / 4} a^{1 / 2}\right)$ and $\left(1-2^{-1 / 4} a^{1 / 2}, 1+2^{-1 / 4} a^{1 / 2}\right)$. Then it follows from (3.3)

$$
e_{-i-2} / e_{-i} \text { and } e_{-i-2}^{\prime} / e_{-i}^{\prime} \text { converge to } c \text { and } c^{\prime} \text { respectively as } i \rightarrow \infty \text {. }
$$

Let $X$ denote the smallest algebraic set in $\mathbf{R}^{2}$ containing the graph of $\tau$. That is of dimension 1 since $\tau$ is semialgebraic. Let $P(x, y)$ be a polynomial whose set of zeros is $X$. Then $P\left(e_{i}, e_{i}^{\prime}\right)=0$ for any $i \in \mathbf{Z}$. Let $P(x, y)=\sum_{i=1}^{l} \delta_{i} x^{\beta_{i}} y^{\gamma_{i}}, \delta_{i} \in \mathbf{R}$ and $\beta_{i}, \gamma_{i} \in \mathbf{N}$, be an expansion. Then we note that $\beta_{i}+\alpha \gamma_{i} \neq \beta_{i^{\prime}}+\alpha \gamma_{i^{\prime}}$ for $i \neq i^{\prime}$, since $\alpha$ is irrational. Set $P_{i}(x, y)=x^{\beta_{i}} y^{\gamma_{i}}$. For any $n \in \mathbf{Z}$, let $D(n)$ denote the $l \times l$-matrix whose $(i, j)$-component is $P_{i}\left(e_{-n-2 j+1}, e_{-n-2 j+1}^{\prime}\right)$. Then

$$
\left(\delta_{1}, \ldots, \delta_{l}\right) D(n)=\left(P\left(e_{-n-1}, e_{-n-1}^{\prime}\right), \ldots, P\left(e_{-n-2 l+1}, e_{-n-2 l+1}^{\prime}\right)\right)=0 .
$$

Particularly, the determinant $|D(n)|=0$. On the other hand we have

$$
|D(n)|=\prod_{i=1}^{l} P_{i}\left(e_{-n-1}, e_{-n-1}^{\prime}\right)\left|D^{\prime}(n)\right|,
$$

where $D^{\prime}(n)$ is the $l \times l$-matrix whose $(i, j)$-component is

$$
P_{i}\left(e_{-n-2 j+1}, e_{-n-2 j+1}^{\prime}\right) / P_{i}\left(e_{-n-1}, e_{-n-1}^{\prime}\right) .
$$

Now (3.4) means that each $(i, j)$-component of $D^{\prime}(n)$ converges to $\left(c^{\beta_{i}} c^{\prime \gamma_{i}}\right)^{j-1}=$ $c^{\left(\beta_{i}+\alpha \gamma_{i}\right)(j-1)}$ as $n \rightarrow \infty$. Hence $\left|D^{\prime}(n)\right|$ converges to a Vandermonde's determinant which equals $\prod_{i<i^{\prime}}\left(c^{\beta_{i^{\prime}}+\alpha \gamma_{i^{\prime}}-c^{\beta_{i}+\alpha \gamma_{i}}}\right) \neq 0$. Therefore $|D(n)| \neq 0$ for large $n$, which is a contradiction. Thus we have proved (3.2) and hence the case $M=\mathbf{R}$.

II. The case $M=\mathbf{R}^{n}, n>1$. Let $E(\varepsilon), \varepsilon>0$, denote the solid open sphere in $\mathbf{R}^{n}$ of radius $\varepsilon$ with center at 0 , and let $p: \mathbf{R}^{n}-0 \rightarrow S^{n-1}=\overline{E(1)}-E(1)$ be the projection such that $p^{-1}(x) \cup 0$ is a ray for any $x \in S^{n-1}$. We use the notations $N_{1}, N_{2}$, etc. defined in I. Set

$$
\begin{gathered}
\theta(x)=|x|-1, \quad N_{1}^{n}=E(1+d), \quad N_{2}^{n}=\mathbf{R}^{n}-\overline{E(1)}, \\
N_{3}^{n}=E(2)-\overline{E(1)}, \quad \overline{N_{3}^{n}}=\overline{E(2)}-E(1) .
\end{gathered}
$$


Then $q=(p, \theta):\left(N_{1}^{n}-0, N_{2}^{n}, \overline{N_{3}^{n}}\right) \rightarrow S^{n-1} \times\left(N_{1}-(-\infty,-1], N_{2}, \bar{N}_{3}\right)$ is a Nash diffeomorphism. Set

$h_{1}^{n}=q^{-1} \circ\left(\right.$ ident $\left.\times h_{1}\right) \circ q: N_{3}^{n} \rightarrow N_{1}^{n}, \quad h_{2}^{n}=q^{-1} \circ\left(\right.$ ident $\left.\times h_{2}\right) \circ q: N_{3}^{n} \rightarrow N_{2}^{n}$.

Let $M_{c}$ be the abstract Nash manifold constructed by the patching $h_{1}^{n}$ and $h_{2}^{n}$. Then (3.1) is trivial.

Let $\rho_{c}^{n}: \overline{N_{3}^{n}} \rightarrow M_{c}$ be the natural map, $0 \in M_{c}$ be the point which corresponds to $0 \in N_{1}^{n}$, and $p_{c}: M_{c}-0 \rightarrow S^{n-1}$ be the projection defined through $q$. For any $z \in S^{n-1}$, define a Nash map $\phi_{c z}^{n}:\left(1-2^{-1 / 4} a^{1 / 2}, 1+2^{-1 / 4} a^{1 / 2}\right) \rightarrow M_{c}$ by

$$
\phi_{c z}^{n}(x)=\rho_{c}^{n} \circ q^{-1}\left(z \times \rho^{-1} \circ \phi_{c}(x)\right) .
$$

Then $\phi_{c z}^{n}$ holds the same properties as $\phi_{c}$ : critical points are all nondegenerate, the set of critical values is $\rho_{c}^{n} \circ q^{-1}(z \times\{0,1\})$, the set of values is $\rho_{c}^{n} \circ q^{-1}(z \times[0,1])$, and $\phi_{c z}^{n}$ vibrates infinitely. Let $0<c, c^{\prime}<1$. Assume $g: M_{c} \rightarrow M_{c^{\prime}}$ is a Nash diffeomorphism. We will prove that $\log c^{\prime} / \log c$ is rational. As $g$ is one-to-one, for any $z \in S^{n-1}$ except at most one point, $\operatorname{im} g \circ \phi_{c z}^{n}$ does not contain $0 \in M_{c^{\prime}}$. It then follows from Remark 2.2 and the infinite vibration property of $\phi_{c z}^{n}$ that $p_{c^{\prime}} \circ g \circ \phi_{c z}^{n}$ is constant (say $\left.z^{\prime}\right)$. Clearly, the images of $q^{-1}\left(z^{\prime} \times N_{2}\right)$ and $q^{-1}\left(z^{\prime} \times\left(N_{1}-(-\infty,-1]\right)\right)$ in $M_{c^{\prime}}$ are affine Nash submanifolds. Hence, $g \circ \rho_{c}^{n} \circ q^{-1}\left(z \times \bar{N}_{3}\right)=\operatorname{img} \circ \phi_{c z}^{n}$ is not contained in the affine Nash submanifolds, which implies that $g \circ \rho_{c}^{n} \circ q^{-1}\left(z \times \bar{N}_{3}\right)$ contains $\rho_{c^{\prime}}^{n} \circ q^{-1}\left(z^{\prime} \times \bar{N}_{3}\right)$. Apply the same argument to $g^{-1}$. Then, $g^{-1} \circ \rho_{c^{\prime}}^{n} \circ$ $q^{-1}\left(z^{\prime} \times \bar{N}_{3}\right)$ contains $\rho_{c}^{n} \circ q^{-1}\left(z \times \bar{N}_{3}\right)$. Therefore,

$$
g \circ \rho_{c}^{n} \circ q^{-1}\left(z \times \bar{N}_{3}\right)=\rho_{c^{\prime}}^{n} \circ q^{-1}\left(z^{\prime} \times \bar{N}_{3}\right) .
$$

Then, in the same way as $\mathrm{I}$, we see that $\log c^{\prime} / \log c$ is rational.

III. The general compact case of $M$. By [5] any compact $C^{\infty}$ manifold in $\mathbf{R}^{m}$ can be approximated by a Nash one in the $C^{\infty}$ topology. Hence we assume that $M$ is an affine Nash manifold containing 0 and contained in $\mathbf{R}^{m}$ and that $T_{0} M^{\perp}$ is transversal to $M$, where $T_{0} M^{\perp}$ is the space of normal vectors of $M$ at 0 in $\mathbf{R}^{m}$. Set $A=T_{0} M^{\perp} \cap M$ (which is a finite set because $A$ is a compact manifold of dimension 0 by the transversality). Let $S^{n-1}, n=\operatorname{dim} M$, be a sphere in the tangent space $T_{0} M$ with center at 0 , and let $r: \mathbf{R}^{m} \rightarrow T_{0} M$ and $p_{1}: T_{0} M-0 \rightarrow S^{n-1}$ be the orthogonal projection and the natural projection, respectively. Set $p=p_{1} \circ r$ on $M-A$. Then $p$ is a Nash map, and there exists an open neighborhood $U$ of 0 in $M$ such that $\left.r\right|_{U}: U \rightarrow r(U)$ is a Nash diffeomorphism. Let $E(\varepsilon)$ be the solid open sphere in $T_{0} M$ of radius $\varepsilon$ with center at 0 . For simplicity we assume $\overline{E(2)} \subset r(U)$. Set

$N_{1}^{\prime n}=r^{-1} E(1+d) \cap U, \quad N_{2}^{\prime n}=M-r^{-1} E(1) \cap U, \quad N_{3}^{\prime n}=r^{-1} E(2) \cap U-\overline{r^{-1} E(1)}$,

and in the same way as II define $h_{1}^{\prime n}: N_{3}^{\prime n} \rightarrow N_{1}^{\prime n}, h_{2}^{\prime n}: N_{3}^{\prime n} \rightarrow N_{2}^{\prime n}$, and an abstract Nash manifold $M_{c}$ by patching $h_{1}^{\prime n}$ and $h_{2}^{\prime n}$. Then the proofs of (3.1) and (3.2) in II remain true in this case except for the statement in II stating that for any $z \in S^{n-1}$, except at most one point, $\operatorname{im} g \circ \phi_{c z}^{n}$ does not contain 0 . We have to replace this by saying that for any $z \in S^{n-1}$, except at most \#A points, im $g \circ \phi_{c z}^{n}$ does not contain $A$. We omit the details.

IV. The general noncompact case of $M$. If $M$ is not compact, we can consider it to be the interior of a compact affine Nash manifold with boundary in $\mathbf{R}^{m}[\mathbf{7}]$. Hence the argument in III holds true. 


\section{REFERENCES}

1. M. Artin and B. Mazur, On periodic points, Ann. of Math. 81 (1965), 82-99.

2. D. R. Chillingworth and J. Hubbard, A note on nonrigid Nash structures, Bull. Amer. Math. Soc. 77 (1971), 429-431.

3. C. G. Gibson, K. Wirthmuller, A. A. du Plessis and E. J. N. Looijenga, Topological stability of smooth mappings, Lecture Notes in Math., vol. 552, Springer, 1976.

4. T. Mostowski, Some properties of the ring of Nash functions, Ann. Scuola Norm. Sup. Pisa Cl. Sci. (4) 3 (1976), 245-266.

5. J. F. Nash, Jr., Real algebraic manifolds, Ann. of Math. 56 (1952), 405-421.

6. R. Palais, Equivariant real algebraic differential topology, Part I: Smoothness categories and Nash manifolds, Notes, Brandeis Univ., 1972.

7. M. Shiota, Classification of Nash manifolds, Ann. Inst. Fourier (Grenoble) 33 (1983), 209-232.

8. M. Shiota and M. Yokoi, Triangulations of subanalytic sets and locally subanalytic manifolds, Trans. Amer. Math. Soc. 286 (1984), 727-750.

Department of Mathematics, College of General Education, Nagoya UniVERSITY, NAGOYA, JAPAN 